\title{
Single-parameter characterization of the thermal equilibrium density profile for intense non-neutral charged particle beams
}

\author{
Ronald C. Davidson and Hong Qin \\ Plasma Physics Laboratory, Princeton University, Princeton, New Jersey 08543
}

(Received 9 June 1999; published 22 November 1999)

\begin{abstract}
The present analysis considers an intense non-neutral ion beam with characteristic axial velocity $V_{b}=\beta_{b} c$ and directed kinetic energy $\left(\gamma_{b}-1\right) m_{b} c^{2}$ propagating in the $z$ direction through an applied focusing field which produces a transverse focusing force $\mathbf{F}_{\text {foc }}=-\gamma_{b} m_{b} \omega_{\beta b}^{2}\left(x \hat{\mathbf{e}}_{x}+y \hat{\mathbf{e}}_{y}\right)$ on a beam ion (smooth focusing approximation). For a thermal equilibrium distribution function $F_{b}\left(\mathcal{H}_{\perp}^{0}\right)=$ const $\times \exp \left(-\mathcal{H}_{\perp}^{0} / T_{b}\right)$, it is shown that the normalized radial density profile $\pi r_{b}^{2} n_{b}^{0}(r) / N_{b}$, plotted as a function of $r / r_{b}$, depends on a single dimensionless parameter, $\delta_{b}=$ $N_{b} Z_{b}^{2} e^{2} / 2 \gamma_{b}^{2} T_{b}$, which is a measure of the normalized beam intensity. Here, $N_{b} \equiv \int d x d y n_{b}^{0}(r)$ is the number of beam ions per unit axial length and $r_{b}^{2} \equiv N_{b}^{-1} \int d x d y r^{2} n_{b}^{0}(r)$ is the mean-square beam radius. "Universal" profiles for the beam density are presented for a wide range of system parameters. The present results are readily extended to the case of a cylindrical non-neutral plasma column confined by a uniform axial magnetic field $B_{0} \hat{\mathbf{e}}_{z}$.
\end{abstract}

PACS numbers: 29.27.Bd, 41.75.-i

A detailed understanding of the influence of spacecharge effects on the equilibrium and stability properties of intense charged particle beams is increasingly important for applications of high-intensity accelerators and transport systems to basic scientific research, heavy ion fusion, spallation neutron sources, waste transmutation, and tritium production [1-6]. In the beam frame, such intense non-neutral beams [1-13] share many properties in common with laboratory-confined non-neutral plasmas [1,14-19], including thermal equilibrium properties, with density profile shape that exhibits a sensitive nonlinear dependence on space-charge intensity [1,2,11-19]. For the case of a thermal equilibrium distribution function $F_{b}\left(\mathcal{H}_{\perp}^{0}\right)$, standard analyses [1,2,11-13] of the nonlinear Vlasov-Maxwell equation for an intense cylindrical beam typically characterize the equilibrium density profile $n_{b}^{0}(r)=\int d x^{\prime} d y^{\prime} F_{b}\left(\mathcal{H}_{\perp}^{0}\right)$ in terms of two parameters corresponding to the (transverse) temperature $T_{b}$ and onaxis density $\hat{n}_{b}$, or scaled versions thereof. Here, $r$ is the radial distance from the beam axis. Indeed, in the earliest $[14]$ and more recent $[1,11,15]$ theoretical analyses of the thermal equilibrium properties of space-charge-dominated beams by Davidson et al., and in thermal equilibrium analyses $[2,13]$ by Reiser et al., the studies have typically investigated equilibrium properties of appropriately normalized quantities as a function of the (dimensionless) onaxis beam intensity $\omega_{p b}^{2} / \omega_{\beta b}^{2}=4 \pi \hat{n}_{b} Z_{b}^{2} e^{2} / \gamma_{b} m_{b} \omega_{\beta b}^{2}$ (proportional to $\hat{n}_{b}$ ) and the characteristic on-axis Debye length $\lambda_{D b}=\left(T_{b} \gamma_{b}^{2} / 4 \pi \hat{n}_{b} Z_{b}^{2} e^{2}\right)^{1 / 2}$ (proportional to $\left.T_{b}^{1 / 2} / \hat{n}_{b}^{1 / 2}\right)$, treating $\hat{n}_{b}$ and $T_{b}$ as independent parameters. The purpose of the present article is to show that the normalized radial profile for $\pi r_{b}^{2} n_{b}^{0}(r) / N_{b}$, plotted versus $r / r_{b}$, can be characterized in terms of a single dimensionless parameter $\delta_{b}$ defined by $\delta_{b}=N_{b} Z_{b}^{2} e^{2} / 2 \gamma_{b}^{2} T_{b}$. Here, $N_{b}=\int d x d y n_{b}^{0}(r)$ is the number of beam ions per unit axial length, $\gamma_{b}$ is the relativistic mass factor, and $r_{b}^{2}=N_{b}^{-1} \int d x d y r^{2} n_{b}^{0}(r)$ is the mean-square beam radius. The fact that the profiles for $\pi r_{b}^{2} n_{b}^{0}(r) / N_{b}$ versus $r / r_{b}$ are "universal" for specified values of $\delta_{b}$ is a very powerful result. For example, a detailed measurement of the radial density profile $n_{b}^{0}(r)$ in thermal equilibrium permits a direct determination of $N_{b}$ and $r_{b}^{2}$, and an inference (through a "best-fit" determination of $\delta_{b}$ ) of the temperature $T_{b}$. The use of detailed measurements of the thermal equilibrium density profile $n_{b}^{0}(r)$ to infer the transverse temperature $T_{b}$ through a best-fit analysis has been employed in recent experimental studies of laboratoryconfined non-neutral plasmas by Chao et al. [19].

In the present paper, following a discussion of the assumptions and theoretical model, the nonlinear VlasovMaxwell equations are investigated analytically and numerically for the case of a thermal equilibrium beam, and universal profiles for $\pi r_{b}^{2} n_{b}^{0}(r) / N_{b}$ are plotted versus $r / r_{b}$. The results are then extended, by analogy, to the case of a rotating, non-neutral plasma column confined by a uniform axial magnetic field $B_{0} \hat{\mathbf{e}}_{z}$ [1,14-19].

The present analysis considers an intense non-neutral ion beam with characteristic radius $r_{b}$ and axial momentum $\gamma_{b} m_{b} \beta_{b} c$ propagating in the $z$ direction. Here, $V_{b}=\beta_{b} c$ is the axial beam velocity, $\gamma_{b}=\left(1-\beta_{b}^{2}\right)^{-1 / 2}$ is the relativistic mass factor, $Z_{b} e$ and $m_{b}$ are the ion charge and rest mass, respectively, and the applied transverse focusing force on a beam ion is modeled (in the smooth focusing approximation) by $\mathbf{F}_{\text {foc }}=-\gamma_{b} m_{b} \omega_{\beta b}^{2}\left(x \hat{\mathbf{e}}_{x}+y \hat{\mathbf{e}}_{y}\right)$, where $(x, y)$ is the transverse displacement from the beam axis and $\omega_{\beta b}=$ const is the focusing frequency. In addition, for present purposes, the particle motion in the beam frame is assumed to be nonrelativistic, and we consider the class of intense non-neutral beam equilibrium solutions $(\partial / \partial t=0)$ to the nonlinear Vlasov-Maxwell equations 
which are axisymmetric $(\partial / \partial \theta=0)$ about the beam axis and are continuous in the axial direction with $\partial / \partial z=0$. Denoting the four-dimensional transverse phase space by $\left(x, y, x^{\prime}, y^{\prime}\right)$, where $x^{\prime}=d x / d s$ and $y^{\prime}=d y / d s$ are (dimensionless) transverse velocities, and $s=\beta_{b} c t$ is the normalized time variable, it is readily shown that distribution functions $f_{b}^{0}\left(x, y, x^{\prime}, y^{\prime}\right)$ of the general form [1,11-13]

$$
f_{b}^{0}=F_{b}\left(\mathcal{H}_{\perp}^{0}\right)
$$

are exact equilibrium solutions to the nonlinear VlasovMaxwell equations. Here, $\mathcal{H}_{\perp}^{0}$ is the single-particle Hamiltonian defined by

$$
\begin{aligned}
\mathcal{H}_{\perp}^{0}= & \frac{1}{2} \gamma_{b} m_{b} \beta_{b}^{2} c^{2}\left(x^{\prime 2}+y^{\prime 2}\right) \\
& +\frac{1}{2} \gamma_{b} m_{b} \omega_{\beta b}^{2}\left(x^{2}+y^{2}\right)+\frac{Z_{b} e}{\gamma_{b}^{2}} \phi^{0}(r),
\end{aligned}
$$

where $r=\left(x^{2}+y^{2}\right)^{1 / 2}$ is the radial distance from the beam axis, and the electrostatic potential $\phi^{0}(r)$ is determined self-consistently in terms of $F_{b}\left(\mathcal{H}_{\perp}^{0}\right)$ from Poisson's equation

$$
\frac{1}{r} \frac{\partial}{\partial r} r \frac{\partial}{\partial r} \phi^{0}(r)=-4 \pi Z_{b} e n_{b}^{0}(r) .
$$

In Eq. (3),

$$
n_{b}^{0}(r)=\int d x^{\prime} d y^{\prime} F_{b}\left(\mathcal{H}_{\perp}^{0}\right)
$$

is the radial number density profile of the beam ions and $\int d x^{\prime} d y^{\prime} \ldots$ denotes $\int_{-\infty}^{\infty} d x^{\prime} \int_{-\infty}^{\infty} d y^{\prime} \ldots$.

Equations (1)-(4) can be used to investigate detailed equilibrium properties in the smooth-focusing approximation for a wide range of choices of equilibrium distribution function $F_{b}\left(\mathcal{H}_{\perp}^{0}\right)[1,11]$. For present purposes, we focus on the particular choice of distribution function $[1,2$, $11-13$ ]

$$
F_{b}\left(\mathcal{H}_{\perp}^{0}\right)=\hat{n}_{b}\left(\frac{\gamma_{b} m_{b} \beta_{b}^{2} c^{2}}{2 \pi T_{b}}\right) \exp \left(-\frac{\mathcal{H}_{\perp}^{0}}{T_{b}}\right),
$$

which corresponds to thermal equilibrium. In Eq. (5), $\hat{n}_{b}$ and $T_{b}$ are positive constants with dimensions of number density and temperature (energy units), respectively. We also assume that the cylindrical conducting wall is far removed from the beam $\left(r_{w} \gg r_{b}\right)$, and without loss of generality we take $\phi^{0}(r=0)=0$. Substituting Eq. (5) into Eq. (4) and carrying out the integrations over $x^{\prime}$ and $y^{\prime}$ readily gives the density profile

$$
n_{b}^{0}(r)=\hat{n}_{b} \exp \left\{-\frac{1}{2 T_{b}}\left[\gamma_{b} m_{b} \omega_{\beta b}^{2} r^{2}+\frac{2 Z_{b} e}{\gamma_{b}^{2}} \phi^{0}(r)\right]\right\},
$$

where the electrostatic potential $\phi^{0}(r)$ is determined selfconsistently from

$$
\frac{1}{r} \frac{\partial}{\partial r} r \frac{\partial}{\partial r} \phi^{0}(r)=-4 \pi Z_{b} e \hat{n}_{b} \exp \left\{-\frac{1}{2 T_{b}}\left[\gamma_{b} m_{b} \omega_{\beta b}^{2} r^{2}+\frac{2 Z_{b} e}{\gamma_{b}^{2}} \phi^{0}(r)\right]\right\} .
$$

In Eqs. (6) and (7), $r^{2}=x^{2}+y^{2}$, and the constant $\hat{n}_{b}=$ $n_{b}^{0}(r=0)$ can be identified with the on-axis beam number density because $\phi^{0}(r=0)=0$ is assumed.

Poisson's equation (7) is a highly nonlinear differential equation for $\phi^{0}(r)$ which must generally be solved numerically, and the corresponding (bell-shaped) density profile determined self-consistently from Eq. (6). Extensive numerical investigations of Eqs. (6) and (7) have been presented in the literature $[1,2,11-17]$ which characterize the equilibrium solutions typically in terms of two parameters corresponding to the temperature $T_{b}$ and the on-axis number density $\hat{n}_{b}$, or scaled versions thereof. The purpose of the present work is to show that appropriately normalized radial profiles for $n_{b}^{0}(r)$ and $\phi^{0}(r)$ can be characterized in terms of a single dimensionless parameter $\delta_{b}$ defined by

$$
\delta_{b}=\frac{N_{b} Z_{b}^{2} e^{2}}{2 \gamma_{b}^{2} T_{b}},
$$

where

$$
N_{b}=\int d x d y d x^{\prime} d y^{\prime} F_{b}\left(\mathcal{H}_{\perp}^{0}\right)=2 \pi \int_{0}^{\infty} d r r n_{b}^{0}(r)
$$

is the number of beam ions per unit axial length. As shown later, the dimensionless parameter $\delta_{b}$ can also be related to the tune depression $\nu_{b} / \nu_{0}$ [see Eq. (16)]. The main point is that a characterization of the solution to Eqs. (6) and (7) by a single dimensionless parameter, $\delta_{b}$ or $\nu_{b} / \nu_{0}$, permits a numerical determination of universal radial profiles, valid over a wide range of values of $N_{b}$ and $T_{b}$.

Even though an exact analytical solution to Eqs. (6) and (7) is not accessible, the key point to recognize is that the entire class of equilibrium solutions to the nonlinear Vlasov-Maxwell equations described by Eq. (1) possess a simple radial force balance constraint [10] that relates the mean-square beam radius, $r_{b}^{2}=\left\langle x^{2}+y^{2}\right\rangle_{0}$, and the unnormalized transverse emittance squared, $\epsilon_{b}^{2}=$ $4\left\langle x^{\prime 2}+y^{\prime 2}\right\rangle_{0}\left\langle x^{2}+y^{2}\right\rangle_{0}$, to the focusing field strength $\left(\omega_{\beta b}^{2}\right)$ and the number of beam ions per unit length $\left(N_{b}\right)$. Here, the statistical average of a phase function $\chi\left(x, y, x^{\prime}, y^{\prime}\right)$ is defined in the usual manner by $\langle\chi\rangle_{0} \equiv$ $N_{b}^{-1} \int d x d y d x^{\prime} d y^{\prime} \chi F_{b}\left(\mathcal{H}_{\perp}^{0}\right)$. Without presenting algebraic details, the equilibrium radial force balance equation is given by [10]

$$
\left(\kappa_{f}-\frac{K_{b}}{2 r_{b}^{2}}\right) r_{b}=\frac{\epsilon_{b}^{2}}{4 r_{b}^{3}},
$$

where the focusing coefficient $\kappa_{f}$ and self-field perveance $K_{b}$ are defined by

$$
\kappa_{f} \equiv \frac{\omega_{\beta b}^{2}}{\beta_{b}^{2} c^{2}}, \quad K_{b} \equiv \frac{2 N_{b} Z_{b}^{2} e^{2}}{\gamma_{b}^{3} m_{b} \beta_{b}^{2} c^{2}} .
$$


Equation (9) is a quadratic equation for $r_{b}^{2}$, and the physically acceptable solution is given by

$$
r_{b}^{2}=r_{\beta}^{2}\left\{\frac{K_{b}}{2 \sqrt{\kappa_{f}} \epsilon_{b}}+\left[\left(\frac{K_{b}}{2 \sqrt{\kappa_{f}} \epsilon_{b}}\right)^{2}+1\right]^{1 / 2}\right\},
$$

where $r_{\beta}^{2} \equiv \epsilon_{b} / 2 \sqrt{\kappa_{f}}$ is the zero-beam-intensity solution $\left(K_{b} \rightarrow 0\right)$ to Eq. (9). For the case considered here, corresponding to the thermal equilibrium distribution in Eq. (5), it is readily shown for arbitrary beam intensity $K_{b}$ that

$$
\epsilon_{b}^{2}=\frac{8 T_{b}}{\gamma_{b} m_{b} \beta_{b}^{2} c^{2}} r_{b}^{2} .
$$

Substituting Eqs. (10) and (12) into Eq. (9) readily gives

$$
r_{b}^{2}=r_{\beta}^{2}\left(1+\delta_{b}\right)
$$

where

$$
r_{\beta}^{2} \equiv \frac{2 T_{b}}{\gamma_{b} m_{b} \omega_{\beta b}^{2}}=\frac{\epsilon_{b}}{2 \sqrt{\kappa_{f}}} .
$$

Equations (11) and (13) are, of course, fully equivalent, leading to the simple identity

$$
\frac{K_{b}}{\sqrt{\kappa_{f}} \epsilon_{b}}=\frac{\delta_{b}}{\left(1+\delta_{b}\right)^{1 / 2}},
$$

where $\delta_{b}=N_{b} Z_{b}^{2} e^{2} / 2 \gamma_{b}^{2} T_{b}$ is defined in Eq. (8). Finally, following Reiser's definition [20], we introduce the effective tune depression $\nu_{b} / \nu_{0}$ defined by [21] $\nu_{b} / \nu_{0} \equiv$ $\left(1-K_{b} / 2 \kappa_{f} r_{b}^{2}\right)^{1 / 2}$. Making use of the definition of $r_{b}^{2}$ in Eq. (11) and the relation between $K_{b} / 2 \sqrt{\kappa_{f}} \epsilon_{b}$ and $\delta_{b}$ in

Eq. (8), it is readily shown that the effective tune depression $\nu_{b} / \nu_{0}$ can be expressed in terms of the dimensionless parameter $\delta_{b}=N_{b} Z_{b}^{2} e^{2} / 2 \gamma_{b}^{2} T_{b}$ by the simple identity

$$
\frac{\nu_{b}}{\nu_{0}}=\frac{1}{\left(1+\delta_{b}\right)^{1 / 2}} \text {. }
$$

Note from Eq. (16) that $\nu_{b} / \nu_{0} \rightarrow 1$ at low beam intensity $\left(\delta_{b} \ll 1\right)$, whereas $\nu_{b} / \nu_{0} \simeq 1 / \delta_{b}^{1 / 2}$ at high beam intensity $\left(\delta_{b} \gg 1\right)$. Comparing Eqs. (15) and (16), we also note that the dimensionless parameter $K_{b} / \sqrt{\kappa_{f}} \epsilon_{b}$ is related to the tune depression $\nu_{b} / \nu_{0}$ by the simple expression

$$
\frac{K_{b}}{\sqrt{\kappa_{f}} \epsilon_{b}}=\frac{\nu_{b}}{\nu_{0}}\left(\frac{\nu_{0}^{2}}{\nu_{b}^{2}}-1\right),
$$

where $\nu_{b} / \nu_{0} \leq 1$. In any case, the dimensionless parameters $\delta_{b}, \nu_{b} / \nu_{0}$, and $K_{b} / \sqrt{\kappa_{f}} \epsilon_{b}$ are all related by the simple expressions in Eqs. (15) -(17), and are equivalent measures of normalized beam intensity.

We now return to the equilibrium Poisson equation (7), and introduce the dimensionless electrostatic potential $\Phi$ and normalized radial coordinate $R$ defined by

$$
\Phi(r) \equiv \frac{Z_{b} e \phi^{0}(r)}{2 \gamma_{b}^{2} T_{b}}, \quad R \equiv \frac{r}{r_{b}},
$$

where the rms beam radius $r_{b}$ is defined in Eq. (11), or equivalently in Eq. (13). Making use of $r_{b}^{2}=r_{\beta}^{2}(1+$ $\delta_{b}$ ), where $r_{\beta}^{2}=2 T_{b} / \gamma_{b} m_{b} \omega_{\beta b}^{2}$, it is readily shown that Eq. (7) can be expressed in the equivalent form

$$
\frac{1}{R} \frac{\partial}{\partial R} R \frac{\partial}{\partial R} \Phi(R)=-8 \delta_{b} \frac{\exp \left\{-\left(1+\delta_{b}\right) R^{2}-\Phi\right\}}{\int_{0}^{\infty} d R^{2} \exp \left\{-\left(1+\delta_{b}\right) R^{2}-\Phi\right\}},
$$

where $R \equiv r / r_{b}$ and $\delta_{b}$ is defined in Eq. (8). Here, use has been made of $N_{b}=2 \pi \int_{0}^{\infty} d r r n_{b}^{0}(r)$ to express the on-axis beam density $\hat{n}_{b}$ as

$$
\hat{n}_{b}=\frac{N_{b}}{\pi r_{b}^{2} \int_{0}^{\infty} d R^{2} \exp \left\{-\left(1+\delta_{b}\right) R^{2}-\Phi\right\}} .
$$

Substituting Eq. (20) into Eq. (6) readily gives for the equilibrium density profile

$$
n_{b}^{0}(r)=\frac{N_{b}}{\pi r_{b}^{2}} \frac{\exp \left\{-\left(1+\delta_{b}\right) r^{2} / r_{b}^{2}-\Phi\right\}}{\int_{0}^{\infty} d R^{2} \exp \left\{-\left(1+\delta_{b}\right) R^{2}-\Phi\right\}} .
$$

The nonlinear Poisson equation (19) is to be solved subject to the boundary conditions $\Phi(R=0)=0=$ $[\partial \Phi / \partial R]_{R=0}$. What is clear from Eq. (19) is that the solution to Eq. (19) for $\Phi(r)$ is characterized by the single dimensionless parameter $\delta_{b}=N_{b} Z_{b}^{2} e^{2} / 2 \gamma_{b}^{2} T_{b}$. For a specified value of $\delta_{b}$, Eq. (19) can be solved numerically for $\Phi(R)$ and the result substituted into Eq. (21) to determine the equilibrium density profile $n_{b}^{0}(r)$. Equation (19) can, of course, be solved analytically in the two limiting cases corresponding to (i) high intensity and low emittance $\left(\delta_{b} \gg 1\right)$ and (ii) low intensity and high emit- tance $\left(\delta_{b} \ll 1\right)$. For example, for $\delta_{b} \gg 1$, it is readily shown that the solution to Eq. (19) in the beam interior is $\Phi \simeq-\delta_{b} R^{2}$, and the corresponding density profile determined from Eq. (21) has the familiar step-function form

$$
n_{b}^{0}(r)= \begin{cases}\frac{N_{b}}{2 \pi r_{b}^{2}}, & 0 \leq r<\sqrt{2} r_{b}, \\ 0, & r>\sqrt{2} r_{b},\end{cases}
$$

where $r_{b}^{2}=r_{\beta}^{2}\left(1+\delta_{b}\right)$. On the other hand, for $\delta_{b} \ll 1$, the solution to Eq. (19) satisfies $|\Phi| \ll R^{2}$ in the beam interior, and the equilibrium density profile determined from Eq. (21) has the Gaussian form

$$
n_{b}^{0}(r)=\frac{N_{b}}{\pi r_{b}^{2}} \exp \left(-r^{2} / r_{b}^{2}\right)
$$

where $r_{b}^{2}=r_{\beta}^{2}\left(1+\delta_{b}\right) \simeq r_{\beta}^{2}$ for $\delta_{b} \ll 1$.

It is clear from Eqs. (21)-(23) that it is convenient to measure the density $n_{b}^{0}(r)$ in units of $N_{b} / \pi r_{b}^{2}$, where $N_{b}$ is the number of ions per unit axial length of the beam and $r_{b}^{2}=r_{\beta}^{2}\left(1+\delta_{b}\right)$ is the mean-square beam radius. Equation (19) has been solved numerically for $\Phi(r)$ for a wide range of values of the dimensionless parameter $\delta_{b}$, and the results substituted into Eq. (21) to determine 
the corresponding self-consistent density profile. The results are summarized in Fig. 1, which presents universal plots of the normalized density $\pi r_{b}^{2} n_{b}^{0}(r) / N_{b}$ versus $r / r_{b}$ for several values of $\delta_{b}$ and the corresponding effective tune depression $\nu_{b} / \nu_{0}=\left(1+\delta_{b}\right)^{-1 / 2}$. The two limiting cases in Fig. 1 with $\delta_{b}=0$ and $\delta_{b} \rightarrow$ $\infty$ correspond, respectively, to the Gaussian profile in Eq. (23) and the step-function profile in Eq. (22). For increasing values of normalized beam intensity $\delta_{b}$, Fig. 1 clearly shows a continuous transition from the Gaussian to step-function profiles. It should also be noted from Eq. (21) that the normalization of all of the curves in Fig. 1 corresponds to $\int_{0}^{\infty} d\left(r^{2} / r_{b}^{2}\right) \pi r_{b}^{2} n_{b}^{0}(r) / N_{b}=1$. For completeness, Fig. 2 shows universal plots versus $\delta_{b}$ of $r_{b} / r_{\beta}$ [Eq. (13) and Fig. 2(a)], $\nu_{b} / \nu_{0}$ [Eq. (16) and Fig. 2(b)], and $\hat{n}_{b} r_{b}^{2} / \hat{n}_{\beta} r_{\beta}^{2}$ [Eq. (20) and Fig. 2(c)]. Here, $r_{\beta}^{2}=2 T_{b} / \gamma_{b} m_{b} \omega_{\beta b}^{2}$ and $\hat{n}_{\beta}=N_{b} / \pi r_{\beta}^{2}$ are the limiting values of mean-square radius and on-axis density for $\delta_{b} \rightarrow 0$.

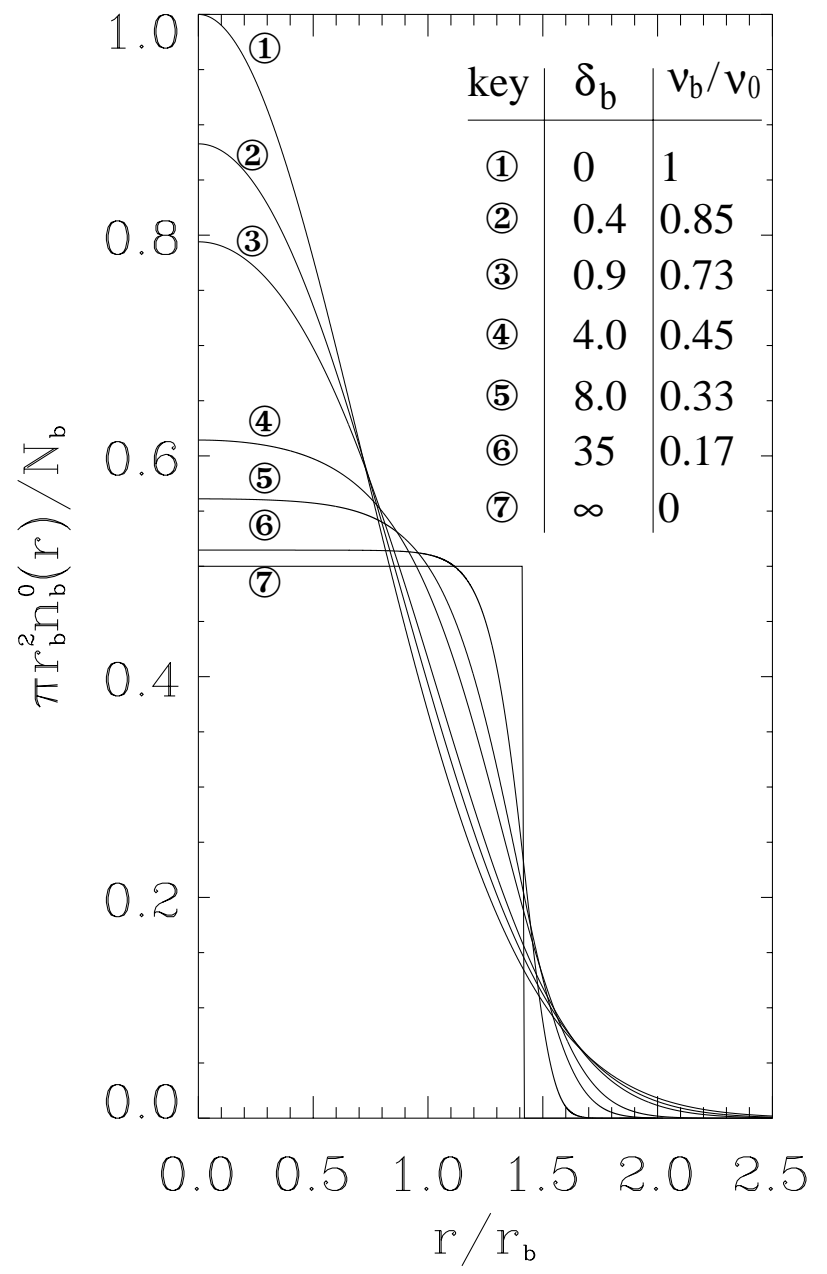

FIG. 1. Plot of normalized density profile $\pi r_{b}^{2} n_{b}^{0}(r) / N_{b}$ versus $r / r_{b}$ for several values of normalized beam intensity $\delta_{b}=N_{b} Z_{b}^{2} e^{2} / 2 \gamma_{b}^{2} T_{b}$ and corresponding values of tune depression $\nu_{b} / \nu_{0}$.
In summary, the fact that the profiles for $\pi r_{b}^{2} n_{b}^{0}(r) / N_{b}$ versus $r / r_{b}$ are universal for specified values of $\delta_{b}$ is a very powerful result. For example, a detailed measurement of the radial density profile $n_{b}^{0}(r)$ in thermal equilibrium permits a direct determination of $N_{b}$ and $r_{b}^{2}$ and an inference (through a best-fit determination of $\delta_{b}$ ) of the
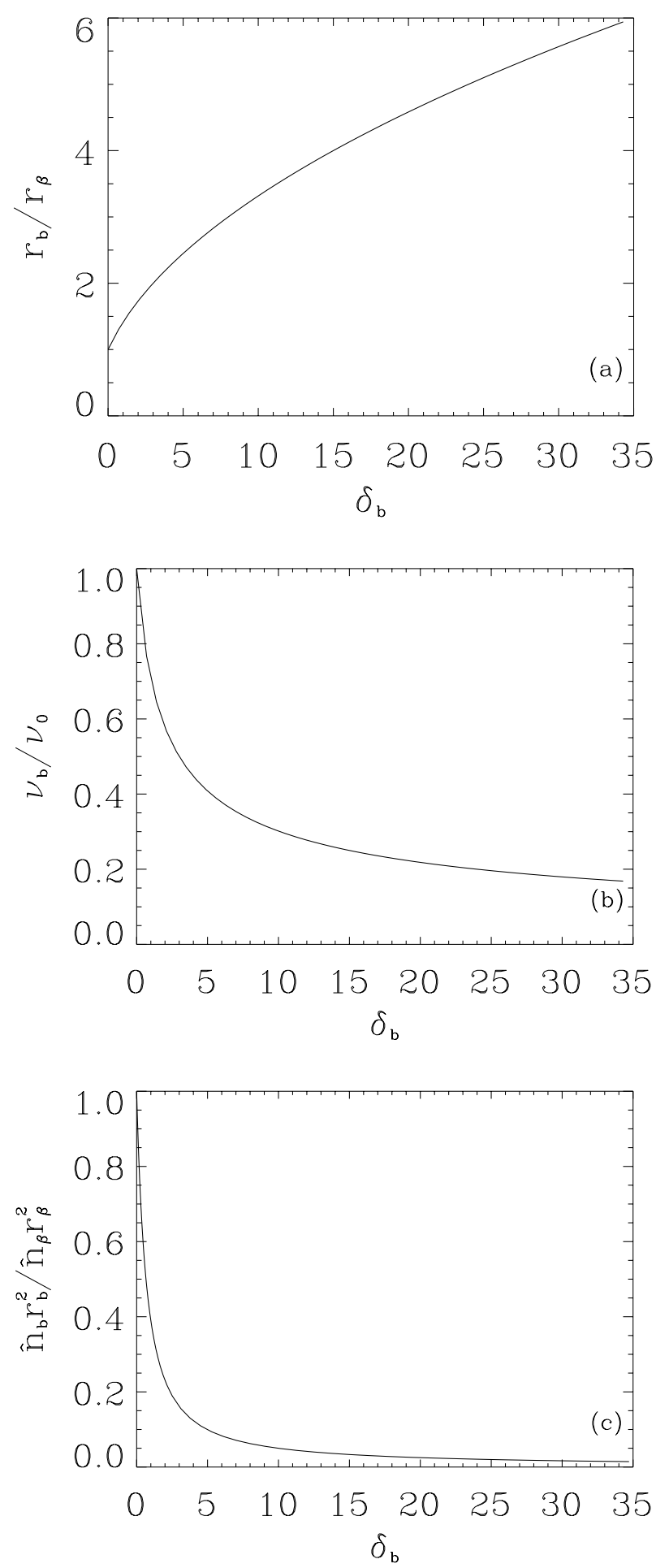

FIG. 2. Plot versus $\delta_{b}=N_{b} Z_{b}^{2} e^{2} / 2 \gamma_{b}^{2} T_{b}$ of (a) $r_{b} / r_{\beta}$ [Eq. (13)], (b) $\nu_{b} / \nu_{0}$ [Eq. (16)], and (c) $\hat{n}_{b} r_{b}^{2} / \hat{n}_{\beta} r_{\beta}^{2}$ [Eq. (20)]. 
temperature $T_{b}$. Furthermore, the present results are readily extended to the case of a nonrelativistic, nonneutral plasma column confined by a uniform axial magnetic field $B_{0} \hat{\mathbf{e}}_{z}$ [14-19]. In this case, we assume the average motion in the $z$ direction is stationary $\left(\beta_{b}=0\right.$ and $\left.\gamma_{b}=1\right)$, and the thermal equilibrium distribution function (in perpendicular phase space) for a plasma column rotating with constant angular velocity $-\omega_{r}$ is given by

$$
F_{b}\left(\mathcal{H}_{\perp}^{0}\right)=\left(\frac{\hat{n}_{b}}{2 \pi m_{b} T_{b}}\right) \exp \left\{-\frac{\mathcal{H}_{\perp}^{0}+\omega_{r} P_{\theta}}{T_{b}}\right\} .
$$

Here, $T_{b}=$ const is the transverse temperature, $\hat{n}_{b}=$ const is the on-axis $(r=0)$ density, $P_{\theta}=r\left[p_{\theta}+\right.$ $\left.\left(Z_{b} e / 2 c\right) r B_{0}\right]$ is the canonical angular momentum, $\mathcal{H}_{\perp}^{0}=\left(2 m_{b}\right)^{-1}\left(p_{r}^{2}+p_{\theta}^{2}\right)+Z_{b} e \phi^{0}(r)$ is the (dimensional) single-particle Hamiltonian, $p_{r}=m_{b} v_{r}$ and $p_{\theta}=$ $m_{b} \boldsymbol{v}_{\theta}$ are the radial and azimuthal components of particle momenta, and the density profile is expressed as $n_{b}^{0}(r)=$ $\int d p_{r} d p_{\theta} F_{b}\left(\mathcal{H}_{\perp}^{0}\right)$, where $N_{b}=2 \pi \int_{0}^{\infty} d r r n_{b}^{0}(r)$ is the number of particles per unit axial length. Some straightforward algebra shows that the resulting equations for the normalized profiles for $\Phi(r)$ and $\pi r_{b}^{2} n_{b}^{0}(r) / N_{b}$ are identical to Eqs. (19) and (21) provided we make the replacements

$$
\begin{aligned}
\Phi(r) \rightarrow \frac{Z_{b} e \phi^{0}(r)}{2 T_{b}}, & \delta_{b} \rightarrow \frac{N_{b} Z_{b}^{2} e^{2}}{2 T_{b}}, \\
\omega_{\beta b}^{2} \rightarrow\left(\omega_{r} \omega_{c b}-\omega_{r}^{2}\right), & r_{\beta}^{2} \rightarrow \frac{\left(2 T_{b} / m_{b}\right)}{\left(\omega_{r} \omega_{c b}-\omega_{r}^{2}\right)},
\end{aligned}
$$

where $\omega_{c b}=Z_{b} e B_{0} / m_{b} c$ is the cyclotron frequency and $r_{b}^{2}=r_{\beta}^{2}\left(1+\delta_{b}\right)$ is the mean-square radius of the plasma column. The angular rotation velocity $-\omega_{r}$ is, of course, related to the average canonical angular momentum $\left\langle P_{\theta}\right\rangle=N_{b}^{-1} \int d x d y d p_{x} d p_{y} P_{\theta} F_{b}\left(\mathcal{H}_{0 \perp}\right)$ by the condition

$$
\left\langle P_{\theta}\right\rangle=m_{b}\left(\omega_{c b} / 2-\omega_{r}\right) r_{b}^{2},
$$

where $\omega_{c b}=Z_{b} e B_{0} / m_{b} c$ is the cyclotron frequency and $r_{b}^{2}$ is the mean-square radius.

Without presenting algebraic details, there are many examples of other equilibrium distribution functions $F_{b}\left(\mathcal{H}_{\perp}^{0}\right)$ for which the normalized equilibrium density profile $\pi r_{b}^{2} n_{b}^{0}(r) / N_{b}$, plotted versus $r / r_{b}$, can be characterized in terms of the single dimensionless normalized intensity parameter $\delta_{b}=N_{b} Z_{b}^{2} e^{2} / 2 \gamma_{b}^{2} T_{b}$. Such examples include the Kapchinskij-Vladimirskij distribution [1,2,11],

$$
F_{b}^{0}\left(\mathcal{H}_{\perp}^{0}\right)=\text { const } \times \delta\left(\mathcal{H}_{\perp}^{0}-T_{b}\right),
$$

and the waterbag distribution $[8,11]$,

$$
F_{b}^{0}\left(\mathcal{H}_{\perp}^{0}\right)=\text { const } \times U\left(\mathcal{H}_{\perp}^{0} / T_{b}\right) .
$$

Here, $T_{b}=$ const, $U(X)$ is the Heaviside step function defined by $U(X)=1$ for $0 \leq X<1$, and $U(X)=0$ for $X>1$. By the same token, there are numerous examples of equilibrium distribution functions for which the normalized equilibrium density profile cannot be characterized in terms of a single dimensionless intensity parameter. Two such examples include the truncated thermal equilibrium distribution function,

$$
F_{b}\left(\mathcal{H}_{\perp}^{0}\right)=\text { const } \times \exp \left(-\mathcal{H}_{\perp}^{0} / T_{b}\right) U\left(\mathcal{H}_{\perp}^{0} / \mathcal{H}_{\perp, \text { max }}\right),
$$

and the truncated inverted population equilibrium distribution function,

$$
\begin{aligned}
F_{b}^{0}\left(\mathcal{H}_{\perp}^{0}\right)= & \text { const } \times\left[1-\left(\mathcal{H}_{\perp}^{0} / \hat{\mathcal{H}}_{\perp}\right)^{2}\right] \\
& \times \exp \left(-\mathcal{H}_{\perp}^{0} / T_{b}\right) U\left(\mathcal{H}_{\perp}^{0} / \mathcal{H}_{\perp, \max }\right) .
\end{aligned}
$$

In Eqs. (29) and (30), $T_{b}, \mathcal{H}_{\perp \text {,max }}$, and $\hat{\mathcal{H}}_{\perp}$ are positive constants, with $\mathcal{H}_{\perp, \max }<\hat{\mathcal{H}}_{\perp}$. For both of the examples in Eqs. (29) and (30), there are additional parameters [ $\mathcal{H}_{\perp, \text { max }}$ in the case of Eq. (29), and $\mathcal{H}_{\perp, \text { max }}$ and $\hat{\mathcal{H}}_{\perp}$ in the case of Eq. (30)] which obviously preclude the singleparameter characterization of the normalized equilibrium density profile $\pi r_{b}^{2} n_{b}^{0}(r) / N_{b}$, plotted versus $r / r_{b}$.

\section{ACKNOWLEDGMENTS}

This research was supported by the Department of Energy and in part by the Office of Naval Research.

[1] R. C. Davidson, Physics of Nonneutral Plasmas (AddisonWesley, Reading, MA, 1990), and references therein.

[2] M. Reiser, Theory and Design of Charged Particle Beams (Wiley, New York, 1994).

[3] T.P. Wangler, Principles of RF Linear Accelerators (Wiley, New York, 1998).

[4] D. A. Edwards and M. J. Syphers, An Introduction to the Physics of High-Energy Accelerators (Wiley, New York, 1993).

[5] R. A. Jameson, in Advanced Accelerator Concepts, edited by J.S. Wurtele, AIP Conf. Proc. No. 279 (AIP, New York, 1993), p. 969.

[6] See, for example, Proceedings of the 12th International Symposium on Heavy Ion Inertial Fusion, edited by I. Hofmann [Nucl. Instrum. Methods Phys. Res., Sect. A 415, 1-725 (1998)].

[7] I. Hofmann, L. J. Laslett, L. Smith, and I. Haber, Part. Accel. 13, 145 (1983).

[8] I. Hofmann and J. Struckmeier, Part. Accel. 21, 69 (1987).

[9] J. Struckmeier and I. Hofmann, Part. Accel. 39, 219 (1992).

[10] R.C. Davidson, W.W. Lee, and P.H. Stoltz, Phys. Plasmas 5, 279 (1998).

[11] R. C. Davidson and C. Chen, Part. Accel. 59, 175 (1998).

[12] R. C. Davidson, Phys. Rev. Lett. 81, 991 (1998).

[13] N. Brown and M. Reiser, Phys. Plasmas 2, 965 (1995).

[14] R. C. Davidson and N. A. Krall, Phys. Fluids 13, 1543 (1970). 
[15] R.C. Davidson and S. M. Lund, in Advances in Plasma Physics, edited by N. J. Fisch, AIP Conf. Proc. No. 314 (AIP, New York, 1994), p. 1.

[16] D. H. E. Dubin and T. M. O’Neil, Rev. Mod. Phys. 71, 20 (1999), and references therein.

[17] T. M. O’Neil and D. H.E. Dubin, Phys. Plasmas 5, 2163 (1998).

[18] T. M. O’Neil, Phys. Today 52(1), 24 (1999).

[19] E. H. Chao, R. C. Davidson, and S. F. Paul, J. Vac. Sci.
Technol. A17, 2050 (1999).

[20] See, for example, p. 216 of Ref. [2].

[21] Strictly speaking, the tune depression [20] defined in Eq. (16) is valid for a uniform density beam with edge radius $\sqrt{2} r_{b}$, whereas the density profile in Eq. (21) varies continuously with radius $r$. For this reason, Eq. (16) should be viewed as a measure of effective tune depression, in an rms sense. 